Pacific Journal of Mathematics

A GEOMETRIC FUNCTION DETERMINED BY EXTREME
POINTS OF THE UNIT BALL OF A NORMED SPACE 


\title{
A GEOMETRIC FUNCTION DETERMINED BY EXTREME POINTS OF THE UNIT BALL OF A NORMED SPACE
}

\author{
RICHARD M. ARON AND ROBERT H. LOHMAN
}

\begin{abstract}
A geometric function, which measures the relative distance of a vector to an extreme point of the unit ball of a normed space, is defined. This function is calculated explicitly for certain classical function and sequence spaces. Radial limits and continuity properties of this function are investigated and some applications are given.
\end{abstract}

Introduction. There are many normed spaces $X$, which are geometrically very different, whose closed unit balls have the following geometric property, called the $\lambda$-property, in common: each member $x$ of the unit ball is a convex combination of an extreme point $e$ of the unit ball and a vector $y$, where $\|y\| \leq 1$ and $e$ is assigned a positive weight. If we vary $e$ and $y$, looking for the "largest possible" weight in such a representation of $x$, we obtain a geometric function of $x$, called the $\lambda$-function, which measures how close $x$ is to being an extreme point of the unit ball. In Section 1 , we make these ideas more precise and calculate explicit formulas for the $\lambda$-function for the classical spaces $C_{X}(T), l_{1}(X), l_{\infty}(X)$ and $c(X)$. It is also shown when the "largest possible" weight is attained in these spaces. Section 2 investigates continuity properties of the $\lambda$-function. These include existence of radial limits (Theorem 2.2) and Lipschitz properties (Corollaries 2.8 and 2.9). In Section 3, it is shown how the uniform $\lambda$-property is related to uniformly convergent series expansions of vectors in terms of infinite convex combinations of extreme points of the unit ball (Theorem 3.1). Local boundedness of the $\lambda$-function away from zero (Theorem 3.5) is also discussed. Section 4 contains a list of questions and open problems.

0. Notation. If $X$ is a normed space, the closed unit ball, open unit ball and unit sphere will be denoted by $B_{X}, U_{X}$ and $S_{X}$, respectively. The symbols $l_{1}(X), l_{\infty}(X)$ and $c(X)$ denote the spaces of all $X$-valued sequences $X=\left(x_{n}\right)$ which are absolutely summable, bounded and convergent, respectively. $l_{1}(X)$ is endowed with the norm $\|x\|=\sum_{n=1}^{\infty}\left\|x_{n}\right\|$, while the norm in $l_{\infty}(X)$ and $c(X)$ is given by $\|x\|=\sup _{n}\left\|x_{n}\right\|$. If $T$ is a 
compact Hausdorff space, $C_{X}(T)$ denotes the space of continuous $X$-valued functions on $T$ endowed with the sup norm. If $x, y \in X$, then $(x: y)$ denotes $\{\lambda x+(1-\lambda) y: 0<\lambda<1\},((x: y]$ has the obvious corresponding meaning). A point $e$ of a convex subset $A$ of $X$ is an extreme point of $A$ if $x, y \in A$ and $e \in(x: y)$ imply $e=x=y$. The set of extreme points of $A$ is denoted by $\operatorname{ext}(A)$. The convex hull of a subset $B$ of $X$ is denoted by $\operatorname{co}(B)$. Recall that $X$ is strictly convex if $\operatorname{ext}\left(B_{X}\right)=S_{X}$. A convex set $A$ is called a polyhedron in case $\operatorname{ext}(A)$ is finite and $A=\operatorname{co}(\operatorname{ext}(A))$. We denote the set of positive integers by $\mathbf{N}$ and, if $X$ is a normed space, $X_{\mathbf{R}}$ denotes $X$ considered as a real vector space. The function $r: X \backslash\{0\} \rightarrow S_{X}$ is defined by $r(z)=z /\|z\|$. If $z, z^{\prime}$ are non-zero vectors, then

$$
\left\|r(z)-r\left(z^{\prime}\right)\right\| \leq 2\left\|z-z^{\prime}\right\| \min \left\{\|z\|^{-1},\left\|z^{\prime}\right\|^{-1}\right\} .
$$

\section{The $\lambda$-property and computation of the $\lambda$-function.}

Definition 1.1. Let $X$ be a normed space and $x \in B_{X}$. If $e \in$ $\operatorname{ext}\left(B_{X}\right),\|y\| \leq 1,0<\lambda \leq 1$ and $x=\lambda e+(1-\lambda) y$, we say the ordered triple $(e, y, \lambda)$ is amenable to $x$. In this case, we define

$$
\lambda(x)=\sup \{\lambda:(e, y, \lambda) \text { is amenable to } x\}
$$

$X$ is said to have the $\lambda$-property if each $x \in B_{X}$ admits an amenable triple. If $X$ has the $\lambda$-property and, in addition, satisfies $\inf \{\lambda(x)$ : $\left.x \in B_{X}\right\}>0$, we say $X$ has the uniform $\lambda$-property.

There are several elementary facts which we record for future use.

Proposition 1.2. Let $X$ be a normed space.

(a) If $e \in \operatorname{ext}\left(B_{X}\right)$, then $\lambda(e)=1$.

(b) If $(e, y, \lambda)$ is amenable to $x$ and $\lambda<1,\|y\|<1$, there exist $\lambda^{\prime}>\lambda$ and $y^{\prime} \in S_{X}$ such that $y \in\left(y^{\prime}: x\right]$ and $\left(e, y^{\prime}, \lambda^{\prime}\right)$ is amenable to $x$.

(c) If $(e, y, \lambda)$ is amenable to $x$ and $0<\lambda^{\prime}<\lambda$, there exists $y^{\prime} \in$ $(y: x)$ such that $\left(e, y^{\prime}, \lambda^{\prime}\right)$ is amenable to $x$.

(d) If $X$ has the $\lambda$-property, then $\lambda(x) \leq(1+\|x\|) / 2$ for all $x \in B_{X}$.

(e) If $X$ is a strictly convex space, then $\lambda(x)=(1+\|x\|) / 2$ for all $x \in B_{X}$ and $\lambda(x)$ is attained.

(f) If $X$ has the $\lambda$-property and $Y$ is a linear subspace of $X$ such that $Y$ has the $\lambda$-property, and $\operatorname{ext}\left(B_{Y}\right) \subset \operatorname{ext}\left(B_{X}\right)$, then $\lambda_{Y}(x) \leq \lambda_{X}(x)$ for all $x$ in $Y$, where $\lambda_{Y}$ and $\lambda_{X}$ are the $\lambda$-functions defined by (2) in $B_{Y}$ and $B_{X}$. 
Proof. (a) This is clear since $(e, e, 1)$ is amenable to $e$.

(b) Since $\lambda<1, x \neq e$ and so there is a $t>0$ such that $y^{\prime}=y+$ $t(x-e)$ has norm one. A straightforward calculation shows that $\lambda^{\prime}=$ $(\lambda+t-t \lambda) /(1+t-t \lambda)$ works.

(c) We may take

$$
y^{\prime}=\frac{\lambda-\lambda^{\prime}}{\lambda\left(1-\lambda^{\prime}\right)} x+\left(1-\frac{\lambda-\lambda^{\prime}}{\lambda\left(1-\lambda^{\prime}\right)}\right) y .
$$

(d) This follows from the fact that if $(e, y, \lambda)$ is amenable to $x$, then $x-\lambda e=(1-\lambda) y$ and so

$$
\lambda-\|x\| \leq\|x-\lambda e\| \leq 1-\lambda .
$$

(e) If $x \in B_{X}$ and $x \neq 0$, then $(x /\|x\|,-x /\|x\|,(1+\|x\|) / 2)$ is amenable to $x$ so that $\lambda(x) \geq(1+\|x\|) / 2$. An appeal to (d) completes the proof. On the other hand, it is clear that $\lambda(0)=1 / 2$.

(f) This is clear from (2).

Before computing the $\lambda$-function for $C_{X}(T)$, we will need the following version of the Borsuk-Dugundji extension theorem.

LEMMA 1.3. Let $T$ be a compact metric space, let $T_{0}$ be a non-empty closed subset of $T$ and let $X$ be an infinite-dimensional normed space. If $g: T_{0} \rightarrow S_{X}$ is a continuous mapping, there exists a continuous mapping $g: T \rightarrow S_{X}$ such that $\left.g\right|_{T_{0}}=g$.

Proof. This follows from Theorem 4.1 and 6.1 of [3] (a considerable strengthening of Theorem 6.1 is found in [1]).

If $e$ is an extreme point of the unit ball of $C_{X}(T)$, one cannot conclude that $e(t) \in \operatorname{ext}\left(B_{X}\right)$ for $t \in T$ (see [2] for a four-dimensional space $X$ in which this fails for $\left.C_{X}([0,1])\right)$. However, all we need here is the following elementary result whose proof is given for the sake of completeness.

LEMMA 1.4. Let $T$ be a compact Hausdorff space and let $X$ be a normed space. If $e$ is an extreme point of the unit ball of $C_{X}(T)$, then $\|e(t)\|=1$ for all $t \in T$.

Proof. Suppose there exists $t_{0} \in T$ such that $\left\|e\left(t_{0}\right)\right\|=\alpha<1$. Let $\delta=(1-\alpha) / 4$ and set $V=\{t \in T: \quad\|e(t)\| \leq \alpha+\delta\}, \quad W=\{t \in T$ : $\|e(t)\| \geq 1-\delta\}$. Then $t_{0} \in V$ and, since $\|e\|=1, W \neq \varnothing$. By Urysohn's 
lemma, there is a continuous function $f: T \rightarrow[0,1]$ such that $f(V)=\{1\}$, $f(W)=\{0\}$. Fix $x_{0} \in S_{X}$ and define $u, v \in C_{X}(T)$ by $u(t)=e(t)+$ $\delta f(t) x_{0}, v(t)=e(t)-\delta f(t) x_{0}$. Then $u, v$ are in the unit ball of $C_{X}(T)$, $u \neq e \neq v$ and $e=(u+v) / 2$, contradicting the fact that $e$ is an extreme point of the unit ball of $C_{X}(T)$.

REMARK 1.5. If $e \in C_{X}(T)$ and $e(t) \in \operatorname{ext}\left(B_{X}\right)$ for all $t \in T$, then $e$ is an extreme point of the unit ball of $C_{X}(T)$. Consequently, if $X$ is a strictly convex normed space, the converse of Lemma 1.4 is true.

THEOREM 1.6. Let $T$ be a compact metric space and let $X$ be an infinite-dimensional strictly convex normed space. Then $C_{X}(T)$ has the uniform $\lambda$-property. In fact, if $x \in C_{X}(T)$ and $\|x\| \leq 1$, then $\lambda(x)=$ $(1+m) / 2$, where $m=\inf \{\|x(t)\|: t \in T\}$. Moreover, if $x(t) \neq 0$ for all $t \in T, \lambda(x)$ is attained.

Proof. Suppose $x=\lambda e+(1-\lambda) y$, where $0 \leq \lambda \leq 1,\|y\|=1$ and $e$ is an extreme point of the unit ball of $C_{X}(T)$. By Lemma 1.4, $\|e(t)\|=1$ for all $t \in T$. Since $\lambda e(t)=x(t)-(1-\lambda) y(t)$, we have

$$
\lambda=\|\lambda e(t)\| \leq\|x(t)\|+(1-\lambda)\|y(t)\| \leq\|x(t)\|+1-\lambda .
$$

Taking the infimum over all $t \in T$ yields $\lambda \leq(1+m) / 2$. Taking the supremum over all such $\lambda$ yields $\lambda(x) \leq(1+m) / 2$.

In order to obtain the reverse inequality, first note that if $m=1$, then $\|x(t)\|=1$ for all $t \in T$. Therefore, $x$ is an extreme point of the unit ball of $C_{X}(T)$ and $\lambda(x)=1=(1+m) / 2$. Hence, we may assume $m<1$. Next, suppose $x(t) \neq 0$ for all $t \in T$. In this case, we define $e, y \in C_{X}(T)$ by

$$
e(t)=\frac{x(t)}{\|x(t)\|}, \quad y(t)=\left[\frac{2\|x(t)\|-1-m}{(1-m)\|x(t)\|}\right] x(t) .
$$

Then $e$ is an extreme point of the unit ball of $C_{X}(T)$. Also, for each $t \in T$ we have

$$
\begin{aligned}
\|y(t)\| & \leq \frac{|2\|x(t)\|-1-m|}{1-m} \\
& \leq \frac{|\|x(t)\|-m|+|||x(t) \|-1|}{1-m} \leq 1
\end{aligned}
$$

and

$$
x=\left(\frac{1+m}{2}\right) e+\left(\frac{1-m}{2}\right) y,
$$

proving $\lambda(x) \geq(1+m) / 2$. 
Finally, suppose $x(t)=0$ for some $t \in T$. Then $(1+m) / 2=1 / 2$ and we want to show $\lambda(x) \geq 1 / 2$. To see this, let $0<\lambda<1 / 2$ and choose $\delta>0$ such that $4 \delta<1-2 \lambda$. Let the closed subset $T_{0}$ of $T$ be defined by

$$
T_{0}=\{t \in T:\|x(t)\| \leq \delta \text { or }\|x(t)\| \geq 2 \delta\} .
$$

Fix $x_{0} \in X,\left\|x_{0}\right\|=1$, and define $e: T_{0} \rightarrow S_{X}$ by

$$
e(t)= \begin{cases}x_{0}, & \text { if }\|x(t)\| \leq \delta, \\ \frac{x(t)}{\|x(t)\|}, & \text { if }\|x(t)\| \geq 2 \delta .\end{cases}
$$

Since $e$ is continuous on $T_{0}$, Lemma 1.3 guarantees that there exists a continuous mapping $e^{\prime}: T \rightarrow S_{X}$ that extends $e . e^{\prime}$ is clearly an extreme point of the unit ball of $C_{X}(T)$. Define $y \in C_{X}(T)$ by

$$
y=\left(x-\lambda e^{\prime}\right) /(1-\lambda) \text {. }
$$

To see that $\|y\| \leq 1$, observe that $\|x(t)\| \geq 2 \delta$ implies

$$
\|y(t)\|=\frac{\|x(t)-\lambda x(t) /\| x(t)\|\|}{1-\lambda}=\frac{|\|x(t)\|-\lambda|}{1-\lambda} \leq 1,
$$

while $\|x(t)\|<2 \delta$ implies

$$
\|y(t)\| \leq \frac{\|x(t)\|+\lambda}{1-\lambda}<\frac{2 \delta+\lambda}{1-\lambda}<\frac{1 / 2}{1-\lambda}<1 .
$$

Since $x=\lambda e^{\prime}+(1-\lambda) y$ and $0<\lambda<1 / 2$ is arbitrary, we have $\lambda(x) \geq$ $1 / 2$.

REMARK 1.7. If $X$ is a finite-dimensional strictly convex space, the conclusion of Theorem 1.6 may fail. In fact, $C_{X}(T)$ may even fail to have the $\lambda$-property. For example, let $T=\{z \in \mathbf{C}:|z| \leq 1\}$ and let $X=\mathbf{C}$. Define $x$ in the unit ball of $C_{\mathbf{C}}(T)$ by $x(z)=z$ for all $z \in T$. Assume $(e, y, \lambda)$ is amenable to $x$. Then $|e(z)|=1$ for all $z \in T$ and $0<\lambda<1$. If $|z|=1$, we have $z=x(z)=\lambda e(z)+(1-\lambda) y(z)$ so that

$$
|z-\lambda e(z)| \leq 1-\lambda \text {. }
$$

This means that $\lambda e(z)$ lies in both and closed disc with center 0 and radius $\lambda$ and the closed disc with center $z$ and radius $1-\lambda$. It follows that $\lambda e(z)=\lambda z$; that is, $e(z)=z$ for all $z$ on the unit circle. The contradiction is reached by observing that $e$ must then be a retract of the unit disc $T$ onto the unit circle. This example clearly generalizes to show that $C_{\mathbf{R}^{n}}(T)$ fails to have the $\lambda$-property if $T$ is the closed unit ball of $\mathbf{R}^{n}$. 
Although Remark 1.7 suggests that infinite dimensionality of $X$ is needed in order to obtain results similar to Theorem 1.6, this is not always the case. If $T$ is given stronger properties, infinite dimensionality of $X$ can be relaxed. To see an example of this, we will need

LeMma 1.8. Let $a<b$ and let $X$ be a normed space satisfying $\operatorname{dim} X_{\mathbf{R}}$ $\geq 2$. If $u, v \in S_{X}$, there exists a continuous function $f:[a, b] \rightarrow S_{X}$ such that $f(a)=u, f(b)=v$. If, in addition, $\|v-u\|<1$, then $f$ may be chosen so that for $t, t^{\prime} \in[a, b]$

$$
\left\|f(t)-f\left(t^{\prime}\right)\right\| \leq \frac{2\left|t-t^{\prime}\right|\|u-v\|}{(b-a)(1-\|u-v\|)^{2}} .
$$

Proof. If $v \neq-u$, then $(1-s) u+s v \neq 0$ for any $s \in[0,1]$. We let $f(t)=r(h(t))$, where

$$
h(t)=\left(\frac{b-t}{b-a}\right) u+\left(\frac{t-a}{b-a}\right) v, \quad a \leq t \leq b .
$$

Then $f:[a, b] \rightarrow S_{X}$ is continuous, $f(a)=u, f(b)=v$ and by (1) $f$ satisfies

$$
\left\|f(t)-f\left(t^{\prime}\right)\right\| \leq 2\left\|h(t)-h\left(t^{\prime}\right)\right\| \min \left\{\|h(t)\|^{-1},\left\|h\left(t^{\prime}\right)\right\|^{-1}\right\} .
$$

If $\|v-u\|<1$, we obtain (3) by observing that

$$
\begin{aligned}
\left\|h(t)-h\left(t^{\prime}\right)\right\| & =\frac{\left|t-t^{\prime}\right|}{b-a}\|u-v\| \text { and } \\
\|h(t)\| & =\left\|u+\frac{t-a}{b-a}(v-u)\right\| \geq 1-\|u-v\| .
\end{aligned}
$$

If $v=-u$, choose $w \in S_{X}, w \neq \pm u$, and let $c=(a+b) / 2$. By the preceding observation, there are continuous functions $f_{1}:[a, c] \rightarrow S_{X}, f_{2}$ : $[c, b] \rightarrow S_{X}$ such that $f_{1}(a)=u, f_{1}(c)=w=f_{2}(c), f_{2}(b)=v$. In this case, combine $f_{1}$ and $f_{2}$ to obtain $f$.

THEOREM 1.9. Let $X$ be a strictly convex normed space satisfying $\operatorname{dim} X_{\mathbf{R}} \geq 2$. Then $C_{X}([0,1])$ has the uniform $\lambda$-property. In fact, if $x \in C_{X}([0,1]),\|x\| \leq 1$ and $m=\inf \{\|x(t)\|: t \in[0,1]\}$, then $\lambda(x)=$ $(1+m) / 2$. Moreover, if $x(t) \neq 0$ for all $t \in[0,1], \lambda(x)$ is attained.

Proof. One proceeds exactly as in the proof of Theorem 1.6, noting that only the case in which $x(t)=0$ for some $t \in T$ needs to be modified. In this case, let $0<\lambda<1 / 2$, choose $\delta>0$ such that $4 \delta<1-2 \lambda$ and let 
the closed subset $T_{0}$ of $[0,1]$ be defined by $T_{0}=T_{1} \cup T_{2}$, where $T_{1}=$ $\{t \in[0,1]:\|x(t)\| \leq \delta\}, T_{2}=\{t \in[0,1]:\|x(t)\| \geq 2 \delta\}$. Fix $x_{0} \in S_{X}$ and define $e: T_{0} \rightarrow S_{X}$ as before. The set $[0,1] \backslash T_{0}$ is a countable disjoint union of open intervals $(a, b)$, where $a, b \in T_{0}$. Extend $e$ to $\tilde{e}:[0,1] \rightarrow S_{X}$, defining $\tilde{e}$ on each such interval $(a, b)$ by $\tilde{e}(t)=f(t)$ for all $t \in(a, b)$, where $f$ is chosen as in Lemma 1.8 with $u=e(a), v=e(b)$. By uniform continuity of $x$ on $[0,1]$, there exists $\eta>0$ such that $\left\|x(t)-x\left(t^{\prime}\right)\right\|<\delta / 2$ whenever $t, t^{\prime} \in[0,1]$ and $\left|t-t^{\prime}\right|<\eta$. Hence, if $t, t^{\prime} \in T_{0}$ and $\left|t-t^{\prime}\right|<$ $\eta$, we have $t, t^{\prime} \in T_{1}$ or $t, t^{\prime} \in T_{2}$. Consequently, the endpoints $a, b$ are both in the same set $T_{1}$ or $T_{2}$ for all but finitely many of the open intervals $(a, b)$. By the uniform continuity of $e$ on $T_{0}$, we may also assume $\left\|e(t)-e\left(t^{\prime}\right)\right\|<1$ whenever $t, t^{\prime} \in T_{0}$ and $\left|t-t^{\prime}\right|<\eta$.

We now show that $\tilde{e}$ is continuous. It is clear that $\tilde{e}$ is continuous at each point of $[0,1] \backslash T_{0}$ and at each point interior to $T_{0}$. If $t_{0}$ is in the boundary of $T_{0}$, then $t_{0}$ is an endpoint, say $a$, of one of the distinguished intervals $(a, b)$ mentioned above. Consequently $\tilde{e}$ is continuous from the right at $t_{0}$. On the other hand, let $\left(t_{n}\right)$ be a sequence in $[0,1]$ such that $t_{n} \uparrow t_{0}$. Since $e$ is continuous at $t_{0}$, we may assume that each $t_{n}$ lies in $[0,1] \backslash T_{0}$. If $t_{0}$ is also a right-hand endpoint of one of the distinguished open intervals whose disjoint union is $[0,1] \backslash T_{0}, \tilde{e}$ is continuous from the left at $t_{0}$. Thus, by taking $n$ sufficiently large, we may assume that $t_{n} \in\left(a_{n}, b_{n}\right)$, where $\left(a_{n}, b_{n}\right)$ is one of the distinguished open intervals, $t_{0}-\eta<a_{n}<b_{n}<t_{0}$ and $a_{n}, b_{n}$ are in the same $T_{i}$. If $t_{0} \in T_{1}$, then $a_{n}, b_{n} \in T_{1}$, which implies $\tilde{e}\left(t_{n}\right)=x_{0}=e\left(t_{0}\right)$ for all $n$. If $t_{0} \in T_{2}$, then $a_{n}, b_{n} \in T_{2}$ and $\left\|e\left(a_{n}\right)-e\left(b_{n}\right)\right\|<1$ for all $n$. By Lemma 1.8, we may assume

$$
\left\|e\left(a_{n}\right)-\tilde{e}\left(t_{n}\right)\right\| \leq \frac{2\left\|e\left(a_{n}\right)-e\left(b_{n}\right)\right\|}{\left(1-\left\|e\left(a_{n}\right)-e\left(b_{n}\right)\right\|\right)^{2}} .
$$

Since $\left\|\tilde{e}\left(t_{0}\right)-\tilde{e}\left(t_{n}\right)\right\| \leq\left\|e\left(t_{0}\right)-e\left(a_{n}\right)\right\|+\left\|e\left(a_{n}\right)-\tilde{e}\left(t_{n}\right)\right\|$, the facts that $e\left(a_{n}\right) \rightarrow e\left(t_{0}\right)$ and $e\left(a_{n}\right)-e\left(b_{n}\right) \rightarrow 0$ imply $\tilde{e}\left(t_{n}\right) \rightarrow e\left(t_{0}\right)$, establishing continuity of $\tilde{e}$. The last part of the proof of Theorem 1.6 now completes the proof.

Remark 1.10. The conclusion of Theorem 1.9 fails if $\operatorname{dim} X_{\mathbf{R}}=1$. In fact, $C_{X}([0,1])$ fails the $\lambda$-property, since in $C_{\mathbf{R}}([0,1])$, the only extreme points of the unit ball are the constant functions \pm 1 . Thus, if we define the unit vector $x \in C_{\mathbf{R}}([0,1])$ by $x(t)=1-2 t$, it is easy to see that there is no triple $(e, y, \lambda)$ amenable to $x$. Also, see Remark 1.7. 
THEOREM 1.11. Let $X$ be a strictly convex normed space. Then $l_{1}(X)$ has the $\lambda$-property but not the uniform $\lambda$-property. In fact, if $x=\left(x_{n}\right) \in$ $l_{1}(X),\|x\| \leq 1$ and $M=\sup \left\{\left\|x_{n}\right\|: n \in \mathbf{N}\right\}$, then

$$
\lambda(x)=(1-\|x\|+2 M) / 2 \text {. }
$$

Moreover, $\lambda(x)$ is attained.

Proof. Suppose $(e, y, \lambda)$ is amenable to $x$. We may assume $\lambda<1$. Write $e=\left(e_{n}\right), y=\left(y_{n}\right)$ and observe that there is a positive integer $m$ such that $e_{m} \in \operatorname{ext}\left(B_{X}\right)$ and $e_{n}=0$ if $n \neq m$. Therefore,

$$
1=\sum_{n \neq m}\left\|y_{n}\right\|+\left\|y_{m}\right\|=\frac{\|x\|-\left\|x_{m}\right\|}{1-\lambda}+\frac{\left\|x_{m}-\lambda e_{m}\right\|}{1-\lambda}
$$

which implies $1-\lambda \geq\|x\|+\lambda-2\left\|x_{m}\right\|$. It follows that

$$
\lambda(x) \leq(1-\|x\|+2 M) / 2 \text {. }
$$

On the other hand, if $x=0$, the result is clear. Hence we may assume $x \neq 0$. In addition, if $x$ has a coordinate $x_{n}$ with $\left\|x_{n}\right\|=1$, then $x$ is an extreme point of the unit ball of $l_{1}(X)$ and the result is clear. Consequently, we assume $\left\|x_{n}\right\|<1$ for all $n$. Pick a positive integer $N$ such that $\left\|x_{N}\right\|=M$. Let $\lambda=(1-\|x\|+2 M) / 2, e=\left(e_{n}\right)$ and $y=\left(y_{n}\right)$, where

$$
\begin{gathered}
e_{n}= \begin{cases}0, & n \neq N, \\
\frac{x_{N}}{\left\|x_{N}\right\|}, & n=N,\end{cases} \\
y_{n}= \begin{cases}\frac{2}{1+\|x\|-2 M} x_{n}, & n \neq N, \\
\frac{\|x\|-1}{(1+\|x\|-2 M) M} x_{n}, & n=N .\end{cases}
\end{gathered}
$$

Then $(e, y, \lambda)$ is amenable to $x$. This shows that $l_{1}(X)$ has the $\lambda$-property, establishes the formula for $\lambda(x)$ and proves that $\lambda(x)$ is attained. In order to see that $l_{1}(X)$ does not have the uniform $\lambda$-property, fix $x^{\prime} \in S_{X}$. If $n$ is any positive integer then the unit vector

$$
x=(\underbrace{x^{\prime} / n, \ldots, x^{\prime} / n, 0}_{n}, 0, \ldots)
$$

of $l_{1}(X)$ satisfies $\lambda(x)=1 / n$.

REMARK 1.12. Only a minor change in notation is required to show that Theorem 1.11 is valid for $\left(\oplus \Sigma_{n} X_{n}\right)_{l_{1}}$, where each $X_{n}$ is a strictly convex normed space. 
THEOREM 1.13. Let $X$ be a strictly convex normed space. Then $l_{\infty}(X)$ has the uniform $\lambda$-property. In fact, if $x=\left(x_{n}\right) \in l_{\infty}(X),\|x\| \leq 1$ and $m=\inf \left\{\left\|x_{n}\right\|: n \in \mathbf{N}\right\}$, then $\lambda(x)=(1+m) / 2$. Moreover, $\lambda(x)$ is attained.

Proof. First, suppose $x_{n_{0}}=0$ for some index $n_{0}$. If $x=\lambda e+$ $(1-\lambda) y$, where $0 \leq \lambda \leq 1, e=\left(e_{n}\right)$ is an extreme point of the unit ball of $l_{\infty}(X)$ and $y=\left(y_{n}\right)$ has norm one, then $\lambda e_{n_{0}}+(1-\lambda) y_{n_{0}}=x_{n_{0}}=0$ implies $\lambda /(1-\lambda)=\left\|y_{n_{0}}\right\| \leq 1$. Thus, $\lambda \leq 1 / 2$ which yields $\lambda(x) \leq 1 / 2$. On the other hand, if $n \neq n_{0}$, then by (e) of Proposition 1.2, we may write $x_{n}=\lambda_{n} e_{n}+\left(1-\lambda_{n}\right) y_{n}$, where $e_{n} \in \operatorname{ext}\left(U_{X}\right),\left\|y_{n}\right\|=1$ and $\lambda_{n}=$ $\left(1+\left\|x_{n}\right\|\right) / 2$. Since $\lambda_{n} \geq 1 / 2$, part (c) of Proposition 1.2 shows that $\left(e_{n}, z_{n}, 1 / 2\right)$ is amenable to $x_{n}$ for some $z_{n} \in B_{X}$. Let

$$
\begin{aligned}
& e=\left(e_{1}, \ldots, e_{n_{0}-1}, e_{n_{0}-1}, e_{n_{0}+1}, e_{n_{0}+2}, \ldots\right), \\
& z=\left(z_{1}, \ldots, z_{n_{0}-1},-e_{n_{0}-1}, z_{n_{0}+1}, z_{n_{0}+2}, \ldots\right) .
\end{aligned}
$$

Then $e$ is an extreme point of the unit ball of $l_{\infty}(X),\|z\|=1$ and $x=\frac{1}{2} e+\frac{1}{2} z$. This, together with $\lambda(x) \leq 1 / 2$, yields $\lambda(x)=1 / 2$ and establishes our assertion in this case. Hence, we may assume $0<\left\|x_{n}\right\| \leq 1$ for all $n$. The assertion is also true if $m=1$ because this implies $x$ is an extreme point of the unit ball of $l_{\infty}(X)$. Thus, we also assume $m<1$. We claim $\lambda(x) \leq(1+m) / 2$. To see this, choose a subsequence $\left(x_{n_{k}}\right)$ of $\left(x_{n}\right)$ such that $\left\|x_{n_{k}}\right\| \rightarrow m$ (in case $\left\|x_{n}\right\|=m$ for some $n$, the claim is proved in a manner similar to what follows). If $e=\left(e_{n}\right)$ is an extreme point of the unit ball of $l_{\infty}(X), y=\left(y_{n}\right)$ has norm at most one, $0 \leq \lambda \leq 1$ and $x=\lambda e+(1-\lambda) y$, then $\lambda<1$, since $\lambda=1$ forces $x=e$ and $m=1$. Then $x_{n_{k}}=\lambda e_{n_{k}}+(1-\lambda) y_{n_{k}}$ implies

$$
\frac{\lambda}{1-\lambda} \leq \frac{\left\|x_{n_{k}}\right\|}{1-\lambda}+\left\|y_{n_{k}}\right\| \leq \frac{\left\|x_{n_{k}}\right\|}{1-\lambda}+1
$$

Letting $k \rightarrow \infty$ yields

$$
\frac{\lambda}{1-\lambda} \leq \frac{m}{1-\lambda}+1
$$

or $\lambda \leq(1+m) / 2$, which proves the claim.

In order to see that $\lambda(x) \geq(1+m) / 2$, let $\lambda=(1+m) / 2, e=\left(e_{n}\right)$, $y=\left(y_{n}\right)$, where

$$
y_{n}=\left\{\begin{array}{cl}
e_{n}=x_{n} /\left\|x_{n}\right\|, & \\
x_{n}, & \left\|x_{n}\right\|=1, \\
\frac{\left(2\left\|x_{n}\right\|-1-m\right)}{(1-m)\left\|x_{n}\right\|} x_{n}, & \left\|x_{n}\right\|<1 .
\end{array}\right.
$$


Then $\|y\| \leq 1$ because if $\left\|x_{n}\right\|<1$, we have

$$
\begin{aligned}
\left\|y_{n}\right\| & =\frac{\left|2\left\|x_{n}\right\|-1-m\right|}{1-m} \\
& \leq \frac{\left|\left\|x_{n}\right\|-m\right|+||\left|x_{n} \|-1\right|}{1-m} \leq \frac{1-m}{1-m}=1 .
\end{aligned}
$$

Since $(e, y, \lambda)$ is amenable to $x$, the proof is complete.

REMARK 1.14 . Only a minor change in notation is required to show that Theorem 1.13 is valid for $\left(\oplus \Sigma_{n} X_{n}\right)_{l_{\infty}}$, where each $X_{n}$ is a strictly convex normed space.

The next result is essentially a corollary to Theorem 1.6. Since, however, $\lambda(x)$ can be attained under more general circumstances than indicated in Theorem 1.6, we present this result as

THEOREM 1.15. Let $X$ be an infinite-dimensional strictly convex normed space. Then $c(X)$ has the uniform $\lambda$-property. In fact, if $x=\left(x_{n}\right) \in c(X)$, $\|x\| \leq 1$ and $m=\inf \left\{\left\|x_{n}\right\|: n \in \mathbf{N}\right\}$, then $\lambda(x)=(1+m) / 2$. Moreover, if $\lim _{n} x_{n} \neq 0$, then $\lambda(x)$ is attained.

Proof. Since $c(X)$ is isometrically isomorphic to $C_{X}(T)$, where $T$ is the one-point compactification of $\mathbf{N}$ when $\mathbf{N}$ has the discrete topology, all of the assertions above, except the last one, follow from Theorem 1.6. In order to complete the proof, write $x_{\infty}=\lim _{n} x_{n}$ and assume $x_{\infty} \neq 0$. We may assume $m<1$; otherwise, $x$ is an extreme point of the unit ball of $c(X)$. If $m>0$, define $e=\left(e_{n}\right), y=\left(y_{n}\right)$ as in (4). Then $e, y \in c(X)$ because $\lim _{n} e_{n}=x_{\infty} /\left\|x_{\infty}\right\|$ and

$$
\lim _{n} y_{n}=\frac{2\left\|x_{\infty}\right\|-1-m}{(1-m)\left\|x_{\infty}\right\|} x_{\infty} \text {. }
$$

As in the proof of Theorem 1.13, $(e, y, \lambda)$ is amenable to $x$, where $\lambda=(1+m) / 2$. Next, suppose $m=0$. Fix $x_{0} \in X,\left\|x_{0}\right\|=1$, and note that the set $D=\left\{n: x_{n}=0\right\}$ is finite. Define $e=\left(e_{n}\right), y=\left(y_{n}\right) \in c(X)$ by

$$
\begin{gathered}
e_{n}= \begin{cases}x_{0}, & n \in D, \\
\frac{x_{n}}{\left\|x_{n}\right\|}, & n \in \mathbf{N} \backslash D,\end{cases} \\
y_{n}= \begin{cases}-x_{0}, & n \in D, \\
\frac{2\left\|x_{n}\right\|-1}{\left\|x_{n}\right\|} x_{n}, & n \in \mathbf{N} \backslash D .\end{cases}
\end{gathered}
$$

Then $(e, y, 1 / 2)$ is amenable to $x$, completing the proof. 
By Theorems 1.11 and 1.13, the dual spaces $l_{1}$ and $l_{\infty}$ have the $\lambda$-property and uniform $\lambda$-property, respectively. Since unit balls of dual spaces are rich in extreme points, one might expect (or at least hope) that dual spaces satisfy the $\lambda$-property. To see that this is not the case in general, let $X=C_{\mathbf{R}}([0,1])^{*}$, which, using the Riesz representation theorem, is identified with the Banach space of regular Borel measures on the Borel subsets of $[0,1]$. If $m$ denotes Lebesgue measure on $[0,1]$, then $m \in B_{X}$. Assume there exists a triple $(e, \mu, \lambda)$ that is amenable to $m$. Then we can write $e= \pm \delta_{t}$ for some $t \in[0,1]$, where $\delta_{t}$ is point evaluation at $t$. If $A=[0,1] \backslash\{t\}$, we obtain $1=m(A)=(1-\lambda) \mu(A)$ or $\|\mu\| \geq \mu(A)$ $=1 /(1-\lambda)>1$, which is a contradiction. Consequently, $C_{\mathbf{R}}([0,1])$ and $C_{\mathbf{R}}([0,1]) *$ both fail to have the $\lambda$-property.

We close this section by showing that all finite-dimensional normed spaces have the uniform $\lambda$-property.

THEOREM 1.16. Let $X$ be a finite-dimensional normed space. Then $X$ has the uniform $\lambda$-property. In fact, if $x \in B_{X}$, then $\lambda(x) \geq 1 /\left(1+\operatorname{dim} X_{\mathbf{R}}\right)$.

Proof. Let $n=\operatorname{dim} X_{\mathbf{R}}$. Then each $x \in B_{X}$ can be written as $x=$ $\sum_{k=1}^{n+1} \lambda_{k} e_{k}$, where $e_{k} \in \operatorname{ext}\left(B_{X}\right), \lambda_{k} \geq 0$ for all $k$ and $\sum_{k=1}^{n+1} \lambda_{k}=1$ (see p. 10 of [4]). There is an index $k_{0}$ with $\lambda_{k_{0}} \geq 1 /(n+1)$. If $\lambda_{k_{0}}=1$, then $x \in \operatorname{ext}\left(B_{X}\right)$ and $\lambda(x)=1$. Otherwise,

$$
\left(e_{k_{0}}, \sum_{k \neq k_{0}} \frac{\lambda_{k}}{1-\lambda_{k_{0}}} e_{k}, \lambda_{k_{0}}\right)
$$

is amenable to $x$, completing the proof.

2. Continuity properties of the $\lambda$-function. The $\lambda$-functions which were explicitly calculated for the classical normed spaces of $\S 1$ are all continuous. However, it is not difficult to construct norms in the plane for which the $\lambda$-function fails to be continuous on $B_{X}$. For example, in $X=\mathbf{R}^{2}$, let $u_{n}, v_{n}$ denote those points having polar coordinates

$$
\left(1, \frac{n \pi}{2(n+1)}\right) \text { and }\left(1, \pi-\frac{n \pi}{2(n+1)}\right)
$$

respectively, for $n=0,1,2, \ldots$ Take $\|\cdot\|$ to be the norm on $X$ whose unit ball is the closed convex hull of all the points $\pm u_{n}$, $\pm v_{n}$. If $w_{n}=\left(u_{n}+u_{n+1}\right) / 2$ and $e$ has polar coordinates $(1, \pi / 2)$, then $e \in$ $\operatorname{ext}\left(B_{X}\right)$ (so that $\left.\lambda(e)=1\right), w_{n} \rightarrow e$ and $\lambda\left(w_{n}\right)=1 / 2$ for all $n$. Although the $\lambda$-function may fail to be continuous at some points of $B_{X}$, it does 
possess some continuity properties in important general cases. Such properties are investigated in this section.

LEMMA 2.1. Let $X$ be a normed space with the $\lambda$-property. If $x \in B_{X}$ and $x \neq 0$, then

$$
\lambda(x) \geq \frac{1+\|x\|}{2} \lambda\left(\frac{x}{\|x\|}\right)
$$

Proof. The assertion is trivially true if $\|x\|=1$, so we assume $\|x\|<1$. Write $z=x /\|x\|, y=-x /\|x\|$. Then $\|z\|=\|y\|=1$ and

$$
x=\left(\frac{1+\|x\|}{2}\right) z+\left(1-\frac{1+\|x\|}{2}\right) y .
$$

Given $\varepsilon>0$, there is a triple $\left(e, y^{\prime}, \lambda\right)$ that is amenable to $z$ for which $\lambda(z)-\varepsilon<\lambda$. Letting

$$
\begin{aligned}
\lambda^{\prime} & =\left(\frac{1+\|x\|}{2}\right) \lambda \text { and } \\
y^{\prime \prime} & =\frac{(1+\|x\|)(1-\lambda) y^{\prime}+(1-\|x\|) y}{2-(1+\|x\|) \lambda},
\end{aligned}
$$

a routine computation shows that $\left(e, y^{\prime \prime}, \lambda^{\prime}\right)$ is amenable to $x$. This shows

$$
\lambda(x) \geq\left(\frac{1+\|x\|}{2}\right)\left(\lambda\left(\frac{x}{\|x\|}\right)-\varepsilon\right)
$$

completing the proof.

THEOREM 2.2. Let $X$ be a normed space satisfying the $\lambda$-property, and let $\tau$ be a Hausdorff vector topology on $X$ which is weaker than the norm topology. If $\operatorname{ext}\left(B_{X}\right)$ is $\tau$-sequentially compact (respectively, $\tau$-compact) and $B_{X}$ is $\tau$-sequentially closed (respectively, $\tau$-closed), then radial limits of $\lambda$ satisfy

$$
\lambda(x)=\lim _{r \rightarrow 1^{-}} \lambda(r x), \quad x \in S_{X} .
$$

Proof. First, assume $\operatorname{ext}\left(B_{X}\right)$ is $\tau$-sequentially compact and $B_{X}$ is $\tau$-sequentially closed. Let $x \in S_{X}$ and $\left(r_{n}\right)$ be a sequence of positive numbers increasing to 1 . It suffices to show $\lambda\left(r_{n} x\right) \rightarrow \lambda(x)$. For each $n \in \mathbf{N}$, there is a triple $\left(e_{n}, y_{n}, \lambda_{n}\right)$ amenable to $r_{n} x$ such that $\lambda\left(r_{n} x\right)-$ $1 / n<\lambda_{n}$. By Lemma 2.1, $\lambda\left(r_{n} x\right) \geq\left[\left(1+r_{n}\right) / 2\right] \lambda(x)$ which implies 
$\lim \inf \lambda\left(r_{n} x\right) \geq \lambda(x)$. Write $\lambda=\lim \sup \lambda\left(r_{n} x\right)$ and choose a subsequence $\left(\lambda_{n_{k}}\right)$ of $\left(\lambda_{n}\right)$ such that $\lambda_{n_{k}} \rightarrow \lambda$. By passing to a subsequence of $\left(e_{n_{k}}\right)$, we may assume there exists $e \in \operatorname{ext}\left(B_{X}\right)$ such that $e_{n_{k}} \rightarrow e$ relative to $\tau$. Then $\lambda_{n_{k}} e_{n_{k}} \rightarrow \lambda e$ relative to $\tau$, which implies $\left(1-\lambda_{n_{k}}\right) y_{n_{k}} \rightarrow x-\lambda e$ relative to $\tau$.

If $\lambda=1$, then $x=e$ and we have

$$
\lambda(x) \leq \liminf \lambda\left(r_{n} x\right) \leq \lim \sup \lambda\left(r_{n} x\right)=\lambda=1=\lambda(x),
$$

implying $\lambda\left(r_{n} x\right) \rightarrow \lambda(x)$.

If $\lambda<1$, then $y_{n_{k}} \rightarrow(x-\lambda e) /(1-\lambda)$ relative to $\tau$. Since $B_{X}$ is $\tau$-sequentially closed, $y=(x-\lambda e) /(1-\lambda) \in B_{X}$. Then $x=\lambda e+$ $(1-\lambda) y$ implies $\lambda(x) \geq \lambda$. Therefore, $\lambda\left(r_{n} x\right) \rightarrow \lambda(x)$.

In case $\operatorname{ext}\left(B_{X}\right)$ is $\tau$-compact and $B_{X}$ is $\tau$-closed, pick $\lambda$ and $\left(\lambda_{n_{k}}\right)$ as before. Then there is a subnet $\left(e_{n_{k \alpha}}\right)$ of $\left(e_{n_{k}}\right)$ and $e \in \operatorname{ext}\left(B_{X}\right)$ such that $e_{n_{k \alpha} .} \rightarrow e$ relative to $\tau$. The argument of the preceding case now applies (using subnets instead of subsequences).

Corollary 2.3. Let $X$ be a Banach space with the $\lambda$-property. Then radial limits of $\lambda$ satisfy

$$
\lambda(x)=\lim _{r \rightarrow 1^{-}} \lambda(r x), \quad x \in S_{X}
$$

in the following cases

(a) $X=Y^{*}$, where $Y$ is a normed space and $\operatorname{ext}\left(B_{X}\right)$ is weak*-sequentially compact (in particular, $\operatorname{dim} X<\infty$ and $\operatorname{ext}\left(B_{X}\right)$ is norm closed).

(b) $X=Y^{*}$, where $Y$ is a normed space, and $\operatorname{ext}\left(B_{X}\right)$ is weak ${ }^{*}$-compact.

REMARK 2.4. If $\operatorname{dim} X<\infty$ and $\operatorname{ext}\left(B_{X}\right)$ is not norm closed, the conclusion of Corollary 2.3 may fail. To see this, let $C^{\prime}$ denote the convex hull of the union of the sets $\{(x, y, 0):|x|,|y| \leq 1\}$ and $\{(x, 0, z)$ : $\left.x^{2}+z^{2}=1, z \geq 0\right\}$ in $\mathbf{R}^{3}$. Let $C=(0,0,1)+C^{\prime}$ and let $\|\cdot\|$ denote the norm on $\mathbf{R}^{3}$ whose unit ball is $B=\operatorname{co}(C \cup-C)$. The unit vector $u=(1,0,1)$, which is not an extreme point of $B$ but is a limit point of the sequence $u_{m}=(\cos m, 0,1+\sin m), m \in \mathbf{N}$, from $\operatorname{ext}(B)$, satisfies $\lambda(u)$ $=1 / 2$ and $\lim _{r \rightarrow 1^{-}} \lambda(r u)=1$.

In order to consider additional results related to continuity of the $\lambda$-function, it is necessary to introduce some auxiliary functions. If $u \in S_{X}$, we let

$$
\lambda(u, x)=\sup \{\lambda: 0 \leq \lambda \leq 1 \text { and } x=\lambda u+(1-\lambda) y
$$

$$
\text { for some } \left.y \in B_{X}\right\}, \quad x \in B_{X} \text {, }
$$


(6) $\alpha(u, x)=\sup \{\alpha: \alpha \geq 0,\|x+\alpha(x-u)\|=1\}, \quad x \in B_{X} \backslash\{u\}$,

$$
y(u, x)=x+\alpha(u, x)(x-u), \quad x \in B_{X} \backslash\{u\} .
$$

Geometrically speaking, if $x \in B_{X} \backslash\{u\}, y(u, x)$ is the unit vector which lies on the line from $u$ through $x$ and is "farthest" from $u$. These functions have some elementary properties which are now stated and whose proofs are left to the reader.

LEMMA 2.5. Let $u \in S_{X}$.

(a) If $x \in B_{X} \backslash\{u\}$, then $\lambda(u, x)=\alpha(u, x) /(1+\alpha(u, x))$.

(b) If $x \in B_{X} \backslash\{u\}$, then $x=\lambda(u, x) u+(1-\lambda(u, x)) y(u, x)$.

(c) If $x \in B_{X} \backslash\{u\}$, then $\lambda(u, x)=\|x-y(u, x)\| /\|u-y(u, x)\|$.

(d) If $X$ has the $\lambda$-property and $x \in B_{X}$, then $\lambda(x)=\sup \{\lambda(e, x)$ : $\left.e \in \operatorname{ext}\left(B_{X}\right)\right\}$.

THEOREM 2.6. Let $X$ be a normed space.

(a) If $\|u\|=1$ and $\|x\|,\|z\|<1$ then

$$
\begin{aligned}
& |\alpha(u, x)-\alpha(u, z)| \\
& \quad \leq\left[\max \left\{\frac{\alpha(u, x)(1+\alpha(u, z))}{1-\|x\|}, \frac{\alpha(u, z)(1+\alpha(u, x))}{1-\|z\|}\right\}\right]\|x-z\| .
\end{aligned}
$$

(b) If $\|u\|=\|v\|=1$ and $\|x\|<1$, then

$$
|\alpha(u, x)-\alpha(v, x)| \leq \frac{\alpha(u, x) \alpha(v, x)}{1-\|x\|}\|u-v\| .
$$

Proof. We provide the details for (a) and note that the proof of (b) is similar. By (7),

$$
y(u, z)=x+\alpha(u, z)(x-u)+(1+\alpha(u, z))(z-x) .
$$

It follows that

$$
\begin{aligned}
& |\|y(u, x)\|-\|x+\alpha(u, z)(x-u)\|| \\
& \quad=|\|y(u, z)\|-\|x+\alpha(u, z)(x-u)\|| \\
& \quad \leq\|y(u, z)-x-\alpha(u, z)(x-u)\| \leq(1+\alpha(u, z))\|x-z\| .
\end{aligned}
$$

We may assume $X$ is a real normed space. Choose $f \in X^{*}$ such that $\|f\|=1$ and

$$
1=f(y(u, x))=f(x)+\alpha(u, x) f(x-u) .
$$


We obtain

$$
f(x-u)=\frac{1-f(x)}{\alpha(u, x)} \geq \frac{1-\|x\|}{\alpha(u, x)} .
$$

By (8),

$$
\begin{aligned}
& (\alpha(u, z)-\alpha(u, x)) f(x-u) \\
& \quad=f(x+\alpha(u, z)(x-u))-f(x+\alpha(u, x)(x-u)) \\
& \quad=f(x+\alpha(u, z)(x-u))-\|y(u, x)\| \\
& \quad \leq\|x+\alpha(u, z)(x-u)\|-\|y(u, x)\| \leq(1+\alpha(u, z))\|x-z\| .
\end{aligned}
$$

An application of (9) to (10) yields

$$
\alpha(u, z)-\alpha(u, x) \leq \frac{\alpha(u, x)(1+\alpha(u, z))}{1-\|x\|}\|x-z\| .
$$

Interchanging the roles of $x$ and $z$ in (11) yields a similar inequality which, when used with (11), produces (a).

COROLlary 2.7. Let $X$ be a normed space.

(a) If $\|u\|=1$ and $\|x\|,\|z\|<1$, then

$$
|\lambda(u, x)-\lambda(u, z)| \leq\left[\max \left\{\frac{\lambda(u, x)}{1-\|x\|}, \frac{\lambda(u, z)}{1-\|z\|}\right\}\right]\|x-z\| .
$$

(b) If $\|u\|=\|v\|=1$ and $\|x\|<1$, then

$$
|\lambda(u, x)-\lambda(v, x)| \leq \frac{\lambda(u, x) \lambda(v, x)}{1-\|x\|}\|u-v\| .
$$

Proof. These both follow from Theorem 2.6 and (a) of Lemma 2.5.

COROllary 2.8. Let $X$ be a normed space and let $0 \leq r<1$. If $\|u\|=\|v\|=1$ and $\|x\|,\|z\| \leq r$, then

$$
|\lambda(u, x)-\lambda(v, z)| \leq \frac{1+r}{2(1-r)}[\|x-z\|+\|u-v\|] .
$$

Consequently, the mapping $(u, x) \rightarrow \lambda(u, x)$ on $S_{X} \times B_{X}$ is a Lipschitz mapping.

Proof. This follows from Corollary 2.7 by observing

$$
|\lambda(u, x)-\lambda(v, z)| \leq|\lambda(u, x)-\lambda(u, z)|+|\lambda(u, z)-\lambda(v, z)|
$$

and that if $\|w\|=1$ and $\|y\| \leq 1$, then $\lambda(w, y) \leq(1+\|y\|) / 2$. 
An immediate consequence of part (d) of Lemma 2.5 and Corollary 2.8 is

COROllary 2.9. Let $X$ be a normed space with the $\lambda$-property, and let $0 \leq r<1$. If $\|x\|,\|z\| \leq r$, then

$$
|\lambda(x)-\lambda(z)| \leq \frac{1+r}{2(1-r)}\|x-z\| .
$$

Consequently, the $\lambda$-function is a Lipschitz mapping on $r B_{X}$ and is continuous on $U_{X}$.

Although the $\lambda$-function is continuous on $U_{X}$ when $X$ is a finite-dimensional normed space, the example of Remark 2.4 shows that points of discontinuity may exist on $S_{X}$. In that particular example, the point of discontinuity $(u=(1,0,1))$ is a limit of extreme points of $B_{X}$ but is not an extreme point. We now show that this situation always leads to points of discontinuity of the $\lambda$-function.

THEOREM 2.10. Let $X$ be a finite-dimensional normed space. If $x$ $\in \overline{\operatorname{ext}\left(B_{X}\right)} \backslash \operatorname{ext}\left(B_{X}\right)$, then $\lambda(x)<1$. Consequently, the $\lambda$-function is not continuous at $x$.

Proof. Assume, to the contrary, that $\lambda(x)=1$. Since $x \notin \operatorname{ext}\left(B_{X}\right)$, there is a triple $\left(e_{1}, y_{1}, \lambda_{1}\right)$ that is amenable to $x$ for which $e_{1} \neq x \neq y_{1}$. Choose $\varepsilon_{1}>0$ such that $e_{1}, y_{1} \notin U\left(x, \varepsilon_{1}\right)$, where $U\left(x, \varepsilon_{1}\right)$ denotes the open ball with center $x$ and radius $\varepsilon_{1}$. Since $\lambda(x)=1$, there exists a triple $\left(e_{2}, y_{2}, \lambda_{2}\right)$ that is amenable to $x$ for which $\lambda_{2}>\max \left\{\lambda_{1},\left(2-\varepsilon_{1}\right) / 2\right\}$. The equality $x=\lambda_{2} e_{2}+\left(1-\lambda_{2}\right) y_{2}$ implies

$$
\left\|x-e_{2}\right\|=\left(1-\lambda_{2}\right)\left\|y_{2}-e_{2}\right\| \leq 2\left(1-\lambda_{2}\right)<\varepsilon_{1} .
$$

Then $x$ is in the relative interior of $\operatorname{co}\left(e_{1}, y_{1}\right)$ in $M_{1}$, the one-dimensional linear manifold containing $x$ and $e_{1}$, and $e_{2} \notin M_{1}$.

Assume that triples $\left(e_{i}, y_{i}, \lambda_{i}\right), 1 \leq i \leq n+1$, amenable to $x$, have been selected, where $\lambda_{1}<\cdots<\lambda_{n+1}<1$, the linear manifold $M_{n}$ containing $x$ and $e_{1}, \ldots, e_{n}$ is $n$ dimensional, $e_{n+1} \notin M_{n}$ and $x$ is in the relative interior of $\operatorname{co}\left(e_{1}, \ldots, e_{n}, y_{1}, \ldots, y_{n}\right)$ in $M_{n}$. Then the linear manifold $M_{n+1}$ containing $x$ and $e_{1}, \ldots, e_{n+1}$ is of dimension $n+1$ and there exists $\varepsilon_{n}>0$ such that $M_{n} \cap U\left(x, \varepsilon_{n}\right) \subset \operatorname{co}\left(e_{1}, \ldots, e_{n}, y_{1}, \ldots, y_{n}\right)$. It follows that there exists $0<\varepsilon_{n+1}<\min _{1 \leq i \leq n}\left\{\left\|x-e_{i}\right\|,\left\|x-y_{i}\right\|\right\}$ such that $M_{n+1} \cap U\left(x, \varepsilon_{n+1}\right) \subset \operatorname{co}\left(e_{1}, \ldots, e_{n+1}, y_{1}, \ldots, y_{n+1}\right)$. Since $\lambda(x)=1$, there 
exists a triple $\left(e_{n+2}, y_{n+2}, \lambda_{n+2}\right)$ amenable to $x$ such that $\lambda_{n+1}<$ $\lambda_{n+2}$ and $\left\|x-e_{n+2}\right\|<\varepsilon_{n+1}$. If $e_{n+2} \in M_{n+1}$, then $e_{n+2} \in$ $\operatorname{co}\left(e_{1}, \ldots, e_{n+1}, y_{1}, \ldots, y_{n+1}\right)$ which implies $e_{n+2}$ is one of the $e_{i}$ 's or $y_{i}$ 's, $1 \leq i \leq n+1$. Since this is impossible by the choice of $\varepsilon_{n+1}$, we obtain $e_{n+2} \notin M_{n+1}$.

By induction, we obtain an increasing sequence $\left(M_{n}\right)$ of linear submanifolds of $X$ such that $M_{n}$ has dimension $n$ for all $n$. Since $\operatorname{dim} X<\infty$, the contradiction establishes $\lambda(x)<1$.

We do not yet know what general conditions will guarantee continuity of $\lambda$ on $B_{X}$. In the simplest of cases (i.e., $B_{X}$ a polyhedron), however, $\lambda$ is well behaved and it is this result we now proceed to demonstrate.

LEMMA 2.11. If $B_{X}$ is a polyhedron and $e \in \operatorname{ext}\left(B_{X}\right)$, then the $\lambda$-function is continuous at $e$.

Proof. Let $e, e_{1}, \ldots, e_{m}$ denote the distinct extreme points of $B_{X}$. There exists $\delta>0$ such that $\|e-y\| \geq \delta$ for $y \in \operatorname{co}\left(e_{1}, \ldots, e_{m}\right)$. Suppose $\left(x_{n}\right)$ is a sequence in $B_{X}$ such that $x_{n} \rightarrow e$. For each $n$, write $x_{n}=\lambda_{n} e+$ $\sum_{k=1}^{m} \lambda_{k_{n}} e_{k}$, where $\lambda_{n}, \lambda_{k_{n}} \geq 0$ and $\lambda_{n}+\sum_{k=1}^{m} \lambda_{k_{n}}=1$. If $\lambda_{n}<1$, then

$$
\begin{aligned}
& y_{n}=\sum_{k=1}^{m} \frac{\lambda_{k_{n}}}{1-\lambda_{n}} e_{k} \in \operatorname{co}\left(e_{1}, \ldots, e_{m}\right) \text { and } \\
& x_{n}=\lambda_{n} e+\left(1-\lambda_{n}\right) y_{n} .
\end{aligned}
$$

In this case, $\left\|x_{n}-e\right\|=\left(1-\lambda_{n}\right)\left\|y_{n}-e\right\| \geq\left(1-\lambda_{n}\right) \delta$. From this, we have $\lambda_{n} \rightarrow 1$ which implies $\lambda\left(x_{n}\right) \rightarrow 1=\lambda(e)$.

REMARK 2.12. Let $B_{X}$ be a polyhedron and let $e_{0}, e_{1}, \ldots, e_{m}$ denote the distinct extreme points of $B_{X}$. If $x \in B_{X}, \lambda\left(e_{0}, x\right)$ is attained. Thus, we may write

$$
x=\lambda\left(e_{0}, x\right) e_{0}+\left(1-\lambda\left(e_{0}, x\right)\right) \sum_{k=0}^{m} \lambda_{k} e_{k},
$$

where $\lambda_{k} \geq 0$ for all $k$ and $\sum_{k=0}^{m} \lambda_{k}=1$. Suppose $x \notin \operatorname{ext}\left(B_{X}\right)$; that is, $\lambda\left(e_{0}, x\right)<1$. If $\lambda_{0}>0$, choose a positive number $\delta$ small enough so that $\lambda\left(e_{0}, x\right)+\delta<1$ and $\delta /\left(1-\lambda\left(e_{0}, x\right)-\delta\right)<\lambda_{0}$. Write $\mu=$ $\delta /\left(1-\lambda\left(e_{0}, x\right)-\delta\right)$. A direct computation shows that if $y$ is taken to be the convex combination

$$
y=\left(\lambda_{0}-\mu\right) e_{0}+\sum_{k=1}^{m} \lambda_{k} e_{k}+\sum_{k=0}^{m} \mu \lambda_{k} e_{k}
$$


of $e_{0}, e_{1}, \ldots, e_{m}$, then $\left(e_{0}, y, \lambda\left(e_{0}, x\right)+\delta\right)$ is amenable to $x$. The contradiction shows $\lambda_{0}=0$. Geometrically, this means that if one considers the line $L$ from $e_{0}$ through $x$, then the unit vector $u$ on $L$ that is farthest from $e_{0}$ lies on a face of $B_{X}$ that does not contain $e_{0}$.

THEOREM 2.13. If $X$ is a finite-dimensional normed space such that $B_{X}$ is a polyhedron, then the $\lambda$-function is continuous on $B_{X}$.

Proof. By Corollary 2.9 and Lemma 2.11, it only remains to show that the $\lambda$-function is continuous at each $x \in S_{X} \backslash \operatorname{ext}\left(B_{X}\right)$. Since $\operatorname{ext}\left(B_{X}\right)$ is finite and $\lambda(\cdot)=\max \left\{\lambda(e, \cdot): e \in \operatorname{ext}\left(B_{X}\right)\right\}$, it suffices to show that each function $\lambda(e, \cdot)$ is continuous at each such $x$. To this end, fix $e \in \operatorname{ext}\left(B_{X}\right)$ and $x \in S_{X} \backslash \operatorname{ext}\left(B_{X}\right)$. Let $\left(x_{n}\right)$ be a sequence in $B_{X}$ such that $x_{n} \rightarrow x$. All of the numbers $\lambda(e, x), \lambda\left(e, x_{n}\right)$ are attained. We first consider two special cases.

Case. I. $\left\|x_{n}\right\|=1$ for all $n$.

We write

$$
\begin{aligned}
x & =\lambda(e, x) e+(1-\lambda(e, x)) y(e, x), \\
x_{n} & =\lambda\left(e, x_{n}\right) e+\left(1-\lambda\left(e, x_{n}\right)\right) y\left(e, x_{n}\right) .
\end{aligned}
$$

Let $\left(\lambda\left(e, x_{n_{k}}\right)\right)$ be any convergent subsequence of $\left(\lambda\left(e, x_{n}\right)\right)$, say $\left(\lambda\left(e, x_{n_{k}}\right)\right)$ converges to $\lambda$. Since $x \neq e$, it follows that $\lambda<1$ and $\left(y\left(e, x_{n_{k}}\right)\right)$ converges to $y=(x-\lambda e) /(1-\lambda)$. Thus, $\|y\|=1$ and $x=$ $\lambda e+(1-\lambda) y$ which implies $0 \leq \lambda \leq \lambda(e, x)$. Assume that $\lambda<\lambda(e, x)$. By (a) of Lemma 2.5, $\alpha\left(e, x_{n_{k}}\right) \rightarrow \alpha$, where $\alpha<\alpha(e, x)$ and $\lambda=$ $\alpha /(1+\alpha)$. Choose $\varepsilon>0$ such that

$$
(\alpha+\varepsilon) /(1+\alpha+\varepsilon)=(\lambda+\lambda(e, x)) / 2 .
$$

For each $k$, write

$$
y_{k}=x_{n_{k}}+\left(\alpha\left(e, x_{n_{k}}\right)+\varepsilon\right)\left(x_{n_{k}}-e\right)=y\left(e, x_{n_{k}}\right)+\varepsilon\left(x_{n_{k}}-e\right) .
$$

If $F_{1}, \ldots, F_{p}$ denote the distinct faces of $B_{X}$, there exist $f_{j} \in X^{*},\left\|f_{j}\right\|=1$, such that $F_{j}=B_{X} \cap f_{j}^{-1}(1), 1 \leq j \leq p$. Moreover, by Remark 2.12 for each $k$, there is a face $F_{j(k)}$ that contains $y\left(e, x_{n_{k}}\right)$ but not $e$. Therefore, $f_{j(k)}(e)<1$ and $f_{j(k)}\left(y\left(e, x_{n_{k}}\right)\right)=1$. Since

$$
x_{n_{k}}-e=\left(1-\lambda\left(e, x_{n_{k}}\right)\right)\left(y\left(e, x_{n_{k}}\right)-e\right),
$$

we obtain from (12) that

$$
\begin{aligned}
f_{j(k)}\left(y_{k}\right) & =f_{j(k)}\left(y\left(e, x_{n_{k}}\right)\right)+\varepsilon f_{j(k)}\left(x_{n_{k}}-e\right) \\
& =1+\varepsilon\left(1-\lambda\left(e, x_{n_{k}}\right)\right)\left(1-f_{j(k)}(e)\right) .
\end{aligned}
$$


By finiteness of the number of faces, we may assume, by (13) and by passing to a subsequence, that there is a common index $j_{0}$ among the $j(k)$ 's satisfying

$$
f_{j_{0}}\left(y_{k}\right)=1+\varepsilon\left(1-\lambda\left(e, x_{n_{k}}\right)\right)\left(1-f_{j_{0}}(e)\right)
$$

for all $k$. Consequently, $\left\|y_{k}\right\| \geq 1+\varepsilon\left(1-\lambda\left(e, x_{n_{k}}\right)\right)\left(1-f_{j_{0}}(e)\right)$ for all $k$. But $y_{k} \rightarrow y+\varepsilon(x-e)=x+(\alpha+\varepsilon)(x-e)$ and $\alpha+\varepsilon<\alpha(e, x)$. This implies $\|y+\varepsilon(x-e)\|=1$ from which we obtain $\lambda\left(e, x_{n_{k}}\right) \rightarrow 1$. The contradiction shows that $\lambda\left(e, x_{n_{k}}\right) \rightarrow \lambda(e, x)$, implying $\lambda\left(e, x_{n}\right) \rightarrow$ $\lambda(e, x)$.

Case II. $\left\|x_{n}\right\|<1$ for all $n$.

The obvious notational modification of the proof of Lemma 2.1 yields

$$
\lambda\left(e, x_{n}\right) \geq \frac{1+\left\|x_{n}\right\|}{2} \lambda\left(e, x_{n} /\left\|x_{n}\right\|\right) \text { for all } n .
$$

Case I then gives $\lambda\left(e, x_{n} /\left\|x_{n}\right\|\right) \rightarrow \lambda(e, x)$. This implies $\lim \inf \lambda\left(e, x_{n}\right) \geq$ $\lambda(e, x)$. On the other hand, the same argument as in the first part of the proof of Case I shows that any cluster point $\lambda$ of $\left(\lambda\left(e, x_{n}\right)\right)$ satisfies $\lambda \leq \lambda(e, x)$. Therefore, $\lim \sup \lambda\left(e, x_{n}\right) \leq \lambda(e, x)$ completing the proof in this case.

The proof of the general case follows from Cases I and II.

\section{Further properties of the $\lambda$-function.}

THEOREM 3.1. Let $X$ be a normed space having the uniform $\lambda$-property. If $0<\lambda<\inf \left\{\lambda(x): x \in B_{X}\right\}$, then for each $x \in B_{X}$, there is a sequence $\left(e_{k}\right)$ in $\operatorname{ext}\left(B_{X}\right)$ such that

$$
\left\|x-\sum_{k=1}^{n} \lambda(1-\lambda)^{k-1} e_{k}\right\| \leq(1-\lambda)^{n}, \quad n=1,2, \ldots
$$

Proof. By (c) of Proposition 1.2, there is a triple $\left(e_{1}, x_{1}, \lambda\right)$ amenable to $x$; that is, $x=\lambda e_{1}+(1-\lambda) x_{1}$. Note that $\left\|x-\lambda e_{1}\right\| \leq 1-\lambda$. By the same reasoning, there is a triple $\left(e_{2}, x_{2}, \lambda\right)$ amenable to $x_{1}$. This yields

$$
x=\lambda e_{1}+\lambda(1-\lambda) e_{2}+(1-\lambda)^{2} x_{2},
$$

and

$$
\left\|x-\lambda e_{1}-\lambda(1-\lambda) e_{2}\right\| \leq(1-\lambda)^{2} .
$$


The sequence $\left(e_{k}\right)$ is obtained by repeating the preceding observations in a simple inductive argument.

REMARK 3.2. If $0<\lambda<1$, then $\sum_{k=1}^{\infty} \lambda(1-\lambda)^{k-1}=1$. Thus, if $X$ has the uniform $\lambda$-property, Theorem 3.1 shows: (a) each $x \in B_{X}$ admits an expansion $\sum_{k=1}^{\infty} \lambda_{k} e_{k}$ as an infinite convex combination of members of $\operatorname{ext}\left(B_{X}\right)$ and (b) the sequences of partial sums of these series converge uniformly for $x \in B_{X}$. It is easy to check that (a) implies $X$ has the $\lambda$-property. Moreover, the converse of Theorem 3.1 holds; that is, if (a) and (b) hold, then $X$ has the uniform $\lambda$-property. To verify the last assertion, note that if (a) and (b) hold, then $X$ (by (a)) has the $\lambda$-property and (by (b)) there is a positive integer $N$ such that if $x \in B_{X}$, we can write $x=\sum_{k=1}^{\infty} \lambda_{k} e_{k}$, where $\left(e_{k}\right)$ is a sequence in $\operatorname{ext}\left(B_{X}\right), \lambda_{k} \geq 0$ for all $k$, $\sum_{k=1}^{\infty} \lambda_{k}=1$ and $\left\|x-\sum_{k=1}^{N} \lambda_{k} e_{k}\right\| \leq 1 / 2$. In particular, if $x \in S_{X}, 1 / 2 \leq$ $\sum_{k=1}^{N} \lambda_{k}$ which implies $1 / 2 N \leq \lambda_{k_{0}}$, for some index $k_{0}$. If $\lambda_{k_{0}}=1, \lambda(x)$ $=1$; if $\lambda_{k_{0}}<1$, then

$$
\left(e_{k_{0}}, \sum_{k \neq k_{0}} \frac{\lambda_{k}}{1-\lambda_{k_{0}}} e_{k}, \lambda_{k_{0}}\right)
$$

is amenable to $x$ and so $\lambda(x) \geq 1 / 2 N$. By Lemma $2.1, \lambda(x) \geq 1 / 4 N$ for all $x \in B_{X}$.

We do not know if the $\lambda$-property implies (a). As the following result shows, however, it does imply a similar but weaker statement.

THEOREM 3.3. Let $X$ be a normed space satisfying the $\lambda$-property.

(i) If a convex function $f: B_{X} \rightarrow \mathbf{R}$ attains its maximum value, then it attains its maximum value at a member of $\operatorname{ext}\left(B_{X}\right)$.

(ii) If $X$ is a Banach space, then $B_{X}$ is the closed convex hull of its set of extreme points.

Proof. (i) Suppose that $f$ attains its maximum value at $x$. Pick a triple $(e, y, \lambda)$ that is amenable to $x$. Since $x=\lambda e+(1-\lambda) y$ and $f$ is a convex function, we have $f(x) \leq \lambda f(e)+(1-\lambda) f(y)$. The fact that $0<\lambda \leq 1$ implies $f(e)=f(x)$.

(ii) Assume, to the contrary, that there exists $x \in B_{X} \backslash \overline{\operatorname{co}\left(\operatorname{ext}\left(B_{X}\right)\right)}$. Then there is a continuous linear functional $f$ on $X_{\mathbf{R}}$ and a number $M$ such that $\|f\|=1$ and $|f(y)| \leq M<f(x)$ for all $y \in \operatorname{co}\left(\operatorname{ext}\left(B_{X}\right)\right)$. By the Bishop-Phelps theorem, there is a continuous linear functional $g$ on $X_{\mathbf{R}}$ such that $\|g\|=1,\|f-g\|<(f(x)-M) / 4$ and $g$ attains its norm on 
$B_{X}$. A straightforward computation shows

$$
|g(y)| \leq M+\frac{f(x)-M}{4}<g(x)
$$

for all $y \in \operatorname{co}\left(\operatorname{ext}\left(B_{X}\right)\right)$. Consequently, $g$ does not attain its maximum value on $B_{X}$ at a member of $\operatorname{ext}\left(B_{X}\right)$. This contradiction of (i) completes the proof.

REMARK 3.4. Recall that a normed space $X$ has the Krein-Milman property if every closed and bounded convex subset of $X$ is the closed convex hull of its set of extreme points. Theorem 3.3 shows that if $X$ satisfies the $\lambda$-property, then $X$ satisfies a restricted version of the Krein-Milman property; namely, $B_{X}$ is the closed convex hull of its set of extreme points. The converse, however, is false. For example, the space $C_{X}(T)$ of Remark 1.7 fails to have the $\lambda$-property. On the other hand, since $X$ is the set of complex numbers, the unit ball of $C_{X}(T)$ is the closed convex hull of its set of extreme points (see [5]).

THEOREM 3.5. Let $X$ be a Banach space with the $\lambda$-property. If $\operatorname{ext}\left(B_{X}\right)$ is countable, then the $\lambda$-function is locally bounded away from 0 in the following sense: Given any $x_{0} \in B_{X}$ and any open neighborhood $W$ of $x_{0}$ in $B_{X}$, there exists a point $x_{0}^{\prime} \in W$, a neighborhood $W^{\prime}$ of $x_{0}^{\prime}$ in $B_{X}$ and $\lambda^{\prime}>0$ such that $\lambda(x) \geq \lambda^{\prime}$ for all $x \in W^{\prime}$.

Proof. Let $\left(e_{n}\right)$ be an enumeration of the members of $\operatorname{ext}\left(B_{X}\right)$ and let $\left(r_{m}\right)$ be an enumeration of the rational numbers in $(0,1)$. If $x \in W$, there is a positive integer $n$ and a triple $\left(e_{n}, y, \lambda\right)$ amenable to $x$. Choosing $m$ such that $r_{m}<\lambda$, there is a triple $\left(e_{n}, y^{\prime}, r_{m}\right)$ amenable to $x$. Consequently, $x \in W_{m n}$, where $W_{m n}=W \cap\left(r_{m} e_{n}+\left(1-r_{m}\right) B_{X}\right)$. This shows that $W=\bigcup_{m, n=1}^{\infty} W_{m n}$ and, since each set $W_{m n}$ is closed in $W$, the Baire category theorem guarantees the existence of indices $m^{\prime}, n^{\prime}$ such that $W_{m^{\prime} n^{\prime}}$ has non-empty interior in $W$. Therefore, there is a point $x_{0}^{\prime} \in W_{m^{\prime} n^{\prime}}$ and $\varepsilon>0$ such that $W_{m^{\prime} n^{\prime}}$ contains $W^{\prime}=W \cap\left\{x \in B_{X}:\left\|x-x_{0}^{\prime}\right\|<\varepsilon\right\}$. It follows that if $x \in W^{\prime}$, we have $\lambda(x) \geq r_{m^{\prime}}$, completing the proof.

If $X$ has the $\lambda$-property and the members of $\operatorname{ext}\left(B_{X}\right)$ are separated, then points in $B_{X}$ that are close to being extreme points of $B_{X}$ possess a unique representation property. In order to make this precise, we need the following. 
Lemma 3.6. Let $X$ be a real normed space with the $\lambda$-property. Assume there is a number $\delta>0$ such that $\left\|e-e^{\prime}\right\| \geq \delta$ whenever $e, e^{\prime} \in \operatorname{ext}\left(B_{X}\right)$ and $e \neq e^{\prime}$. If $x \in B_{X}$ and $(e, y, \lambda),\left(e^{\prime}, y^{\prime}, \lambda^{\prime}\right)$ are amenable to $x$, where $\lambda, \lambda^{\prime}>3 /(3+\delta)$, then $e=e^{\prime}$.

$$
\text { Proof. We have } x=\lambda e+(1-\lambda) y=\lambda^{\prime} e^{\prime}+\left(1-\lambda^{\prime}\right) y^{\prime} \text { and so }
$$

$$
\lambda\left(e-e^{\prime}\right)=\left(\lambda^{\prime}-\lambda\right) e^{\prime}-(1-\lambda) y+\left(1-\lambda^{\prime}\right) y^{\prime} .
$$

If $e \neq e^{\prime}$, then (14) and the fact that $\left\|e-e^{\prime}\right\| \geq \delta$ imply

$$
\begin{aligned}
\frac{3 \delta}{3+\delta} & <\lambda\left\|e-e^{\prime}\right\| \leq\left|\lambda^{\prime}-\lambda\right|+2-\lambda-\lambda^{\prime} \\
& <3\left(1-\frac{3}{3+\delta}\right)=\frac{3 \delta}{3+\delta} .
\end{aligned}
$$

The contradiction shows $e=e^{\prime}$.

THEOREM 3.7. Let $X$ be a real normed space with the $\lambda$-property. Assume there is a number $\delta>0$ such that $\left\|e-e^{\prime}\right\| \geq \delta$ whenever $e, e^{\prime} \in$ $\operatorname{ext}\left(B_{X}\right)$ and $e \neq e^{\prime}$. If $x \in B_{X} \backslash \operatorname{ext}\left(B_{X}\right)$ and $\lambda(x)>3 /(3+\delta)$, then there exists a unique pair of vectors $e \in \operatorname{ext}\left(B_{X}\right), y \in S_{X}$ such that $x=$ $\lambda(x) e+(1-\lambda(x)) y$.

Proof. Let $\left(e_{n}, y_{n}, \lambda_{n}\right)$ be a sequence of triples that are amenable to $x$ and for which $\lambda_{n} \uparrow \lambda(x), \lambda_{n}>3 /(3+\delta)$ for all $n$ and $\left\|y_{n}\right\|=1$ for all $n$. By Lemma 3.6, all the $e_{n}$ 's are equal, say to $e$. Since $x=\lambda_{n} e+\left(1-\lambda_{n}\right) y_{n}$ for all $n$, we have $\left(1-\lambda_{n}\right) y_{n} \rightarrow x-\lambda(x) e$. Also, $x \neq e$ implies $\lambda(x)<1$. Thus, if we let $z=x-\lambda(x) e,\|z\|=1-\lambda(x)$. Letting $y=z /\|z\|$ shows $x=\lambda(x) e+(1-\lambda(x)) y$, proving existence. If we also have $x=\lambda(x) e^{\prime}$ $+(1-\lambda(x)) y^{\prime}$, wher $e^{\prime} \in \operatorname{ext}\left(B_{X}\right)$ and $\left\|y^{\prime}\right\|=1$, Lemma 3.6 implies $e=e^{\prime}$. Since $1-\lambda(x) \neq 0$, we also obtain $y=y^{\prime}$.

4. Questions and open problems. The following list of questions is not meant to be exhaustive. Rather, it represents those questions which are of most interest to the authors.

1. It would be useful to calculate the $\lambda$-function for other classical spaces with the $\lambda$-property. In particular, what spaces of operators have the $\lambda$-property and what does the $\lambda$-function look like for these spaces? 
2. If $\left(X_{n}\right)$ is a sequence of normed spaces, each having the $\lambda$-property, when do $\left(\oplus \sum_{n=1}^{\infty} X_{n}\right)_{l_{1}},\left(\oplus \sum_{n=1}^{\infty} X_{n}\right)_{l_{\infty}}$ have the $\lambda$-property and what do their $\lambda$-functions look like?

3. If $X$ is a normed space having the $\lambda$-property, characterize the points of continuity on $S_{X}$ of the $\lambda$-function. Characterize those $x \in B_{X}$ for which $\lambda(x)$ is attained.

4. If $X$ is a Banach space, is the $\lambda$-function of the first Baire class on $B_{X}$ ?

5. If $X$ is a normed space having the $\lambda$-property, can $X$ be renormed so as to have the uniform $\lambda$-property?

6. If a normed space $X$ has the $\lambda$-property, does $X^{*}$ have the $\lambda$-property? In considering the converse, note that $l_{1}$ has the $\lambda$-property and $c_{0}$, one of its preduals, does not. However, $c$ is a predual of $l_{1}$ that has the $\lambda$-property.

\section{REFERENCES}

[1] Y. Benyamini, and Y. Sternfeld, Spheres in infinite-dimensional normed spaces are Lipschitz contractible, Proc. Amer. Math. Soc., 88 (1983), 439-445.

[2] R. M. Blumenthal, J. Lindenstrauss and R. R. Phelps, Extreme operators into $C(K)$, Pacific J. Math., 15 (1965), 747-756.

[3] J. Dugundji, An extension of Tietze's theorem, Pacific J. Math., 1 (1951), 353-367.

[4] R. R. Phelps, Lectures on Choquet's Theorem, Van Nostrand Math. Studies, \#7, 1966, Princeton.

[5] , Extreme points in function algebras, Duke Math. J., 32 (1965), 267-278.

Received December 18, 1985 and in revised form April 2, 1986.

Kent State UNIVERSITY

KENT, OH 44242 



\section{PACIFIC JOURNAL OF MATHEMATICS EDITORS}

\author{
V. S. VARADARAJAN \\ (Managing Editor) \\ University of California \\ Los Angeles, CA 90024 \\ HeRbert Clemens \\ University of Utah \\ Salt Lake City, UT 84112 \\ R. FINN \\ Stanford University \\ Stanford, CA 94305
}

\author{
HERMANN FLASCHKA \\ University of Arizona \\ Tucson, AZ 85721 \\ RAMESH A. GANGOLLI \\ University of Washington \\ Seattle, WA 98195 \\ VAUGHAN F. R. JONES \\ University of California \\ Berkeley, CA 94720 \\ ROBION KIRBY \\ University of California \\ Berkeley, CA 94720
}

\author{
C. C. MOORE \\ University of California \\ Berkeley, CA 94720 \\ H. SAMELSON \\ Stanford University \\ Stanford, CA 94305 \\ HAROLD STARK \\ University of California, San Diego \\ La Jolla, CA 92093
}

\section{ASSOCIATE EDITORS}
R. Arens
E. F. BECKENBACH
B. H. NEUMANN
F. WOLF
K. YosHIDA (1906-1982)

\section{SUPPORTING INSTITUTIONS}

UNIVERSITY OF ARIZONA
UNIVERSITY OF BRITISH COLUMBIA
CALIFORNIA INSTITUTE OF TECHN
UNIVERSITY OF CALIFORNIA
MONTANA STATE UNIVERSITY
UNIVERSITY OF NEVADA, RENO
NEW MEXICO STATE UNIVERSITY
OREGON STATE UNIVERSITY

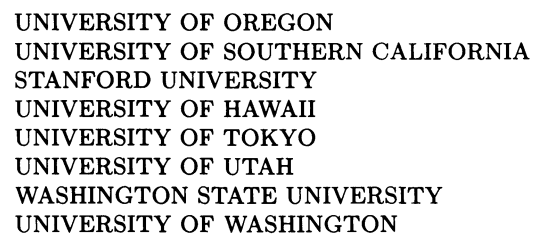

The Supporting Institutions listed above contribute to the cost of publication of this Journal, but they are not owners or publishers and have no responsibility for its content or policies.

Mathematical papers intended for publication in the Pacific Journal of Mathematics should be in typed form or offset-reproduced (not dittoed), double spaced with large margins. Please do not use built up fractions in the text of the manuscript. However, you may use them in the displayed equations. Underline Greek letters in red, German in green, and script in blue. The first paragraph must be capable of being used separately as a synopsis of the entire paper. In particular it should contain no bibliographic references. Please propose a heading for the odd numbered pages of less than 35 characters. Manuscripts, in triplicate, may be sent to any one of the editors. Please classify according to the scheme of Math. Reviews, Index to Vol. 39. Supply name and address of author to whom proofs should be sent. All other communications should be addressed to the managing editor, or Elaine Barth, University of California, Los Angeles, California 90024.

There are page-charges associated with articles appearing in the Pacific Journal of Mathematics. These charges are expected to be paid by the author's University, Government Agency or Company. If the author or authors do not have access to such Institutional support these charges are waived. Single authors will receive 50 free reprints; joint authors will receive a total of 100 free reprints. Additional copies may be obtained at cost in multiples of 50 .

The Pacific Journal of Mathematics is issued monthly as of January 1966. Regular subscription rate: $\$ 190.00$ a year (5 Vols., 10 issues). Special rate: $\$ 95.00$ a year to individual members of supporting institutions.

Subscriptions, orders for numbers issued in the last three calendar years, and changes of address should be sent to Pacific Journal of Mathematics, P.O. Box 969, Carmel Valley, CA 93924, U.S.A. Old back numbers obtainable from Kraus Periodicals Co., Route 100, Millwood, NY 10546.

The Pacific Journal of Mathematics at P.O. Box 969, Carmel Valley, CA 93924 (ISSN 0030-8730) publishes 5 volumes per year. Application to mail at Second-class postage rates is pending at Carmel Valley, California, and additional mailing offices. Postmaster: send address changes to Pacific Journal of Mathematics, P.O. Box 969, Carmel Valley, CA 93924.

PUBLISHED BY PACIFIC JOURNAL OF MATHEMATICS, A NON-PROFIT CORPORATION Copyright (C) 1987 by Pacific Journal of Mathematics 


\section{Pacific Journal of Mathematics \\ Vol. 127, No. $2 \quad$ February, 1987}

Richard Martin Aron and Robert Henry Lohman, A geometric function determined by extreme points of the unit ball of a normed space .... . . 209

Kari Astala and M. S. Ramanujan, $(s)$-nuclear sets and operators $\ldots \ldots \ldots 233$ Earl Robert Berkson, Thomas Alastair Gillespie and Paul Scott Muhly, Analyticity and spectral decompositions of $L^{p}$ for compact abelian

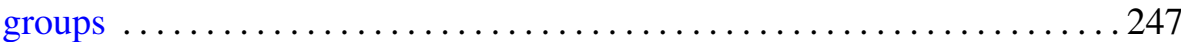

Ronald P. Brown, Real closures of fields at orderings of higher level . . . . . 261

Ronald P. Brown, The behavior of chains of orderings under field

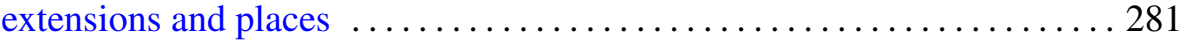

Rodney Graham Downey, Subsets of hypersimple sets .............. 299

Manfred Droste and Saharon Shelah, On the universality of systems of

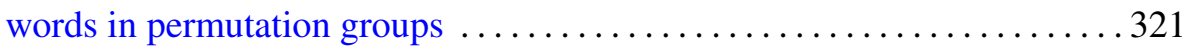

Hidenori Fujiwara, Représentations monomiales des groupes de Lie

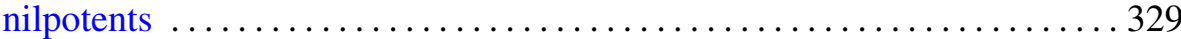

Rupert Lasser, Convolution semigroups on hypergroups $\ldots \ldots \ldots \ldots \ldots 35$

Marcus Marlene Marsh, $u$-mappings on trees $\ldots \ldots \ldots \ldots \ldots \ldots \ldots \ldots \ldots \ldots$

Peter Andrew Symonds, Localization in the classification of flat

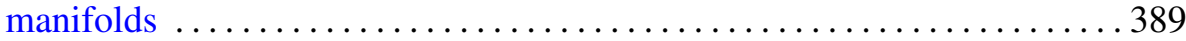

